\title{
Resultado del tratamiento endoscópico con Deflux en el reflujo vesicoureteral primario
}

\author{
Escala Aguirre JM, Retamal Pinto G, Cadena González Y, López Egaña PJ, Letelier Cancino N, \\ Zubieta Acuña R.
}

Unidad de Urología. Servicio de Cirugía Infantil. Hospital Exequiel González Cortes. Santiago-Chile.

Actas Urol Esp. 2007;31(8):880-884

\section{RESUMEN}

\section{RESULTADO DEL TRATAMIENTO ENDOSCÓPICO CON DEFLUX EN EL REFLUJO VESICOURETERAL PRIMARIO}

Propósito: El tratamiento endoscópico en el reflujo vésicoureteral primario es una alternativa disponible desde hace ya un tiempo. En nuestro hospital estamos usando el Deflux (copolímero de dextranomero en acido hialurónico) desde 2002. En éste trabajo revisamos la efectividad de éste procedimiento en pacientes con distintos tipos y grados de reflujo.

Método: Analizamos en forma prospectiva 25 pacientes, con 38 uréteres refluyentes, inyectados desde Enero 2002 hasta Diciembre 2005 que contaran con ecografia y uretrocistografia post operatoria.

Resultados: El 86\% (21) eran de sexo femenino, y su edad promedio fue de 6,1 años (2 a 14 a). El resultado fue exitoso en un 73,6\% ( $n=28)$ de los casos con una inyección, la cantidad de sustancia inyectada no fue relevante en el resultado. Se obtuvo mucho mejor resultado en los grados bajos, alcanzando un 100\% de resolución en los grados 1 y $2(n=15)$ al igual que en los casos de uréter simple y unilateral $(n=10)$. El peor resultado fue obtenido en los casos de uréter doble, resolviéndose solo en 1 de 6 uréteres. El procedimiento fue siempre ambulatorio y no tuvimos complicaciones perioperatorias.

Conclusiones: Nos parece que el tratamiento endoscópico con Deflux es un muy buen tratamiento para el reflujo vésicoureteral primario como alternativa tanto al tratamiento médico, como al quirúrgico, especialmente en los casos unilaterales y en sistemas únicos. Los autores recomiendan esta técnica en el momento de aconsejar a los padres.

Palabras clave: Reflujo vésico ureteral, Endoscópico.

ABSTRACT

ENDOSCOPIC TREATMENT WITH DEFLUX FOR PRIMARY VESICOURETERAL REFLUX

Purpose: Effectivety for endoscopic treatment for primary reflux has been under discussion as a single procedure. In the last 3 years our unit have been used Deflux, (dextranomer copolymer in hialuronic acid) for this pathology. The aim of this study is to analyze the results of our experience

Material and methods: Since 2002, a prospective protocol for VUR has been applied. We reviewed the last 25 cases treated with Deflux\%o injection who had ultrasound and cistography.

Results: $86 \%(n=21)$ were females and with a mean age of 6.1 years (range 2-14) the success rate with a single injection was $73.6 \%(\mathrm{n}=28)$. The amount of deflux injected was irrelevant in the result . The results in the low grades reflux (I-II) reaching the $100 \%(n=15)$. The worse result was in the double system cases with just one successful case out of 6 injected. The procedure was in outpatient bases. There were no periprocedures complications.

Conclusions: The endoscopic treatment for VUR with Deflux, is a good alternative to medical treatment especially in single ureter with low grade. Therefore the authors recommend this technique at the time of counseling parents.

Keywords: Endoscopic. Vésico ureteral reflux. 
$\mathrm{E}^{1}$ reflujo vésico ureteral (RVU) se encuentra en alrededor del 1\% de la población, se asocia frecuentemente a infección urinaria febril y puede llevar a insuficiencia renal crónica, de allí la importancia de su tratamiento ${ }^{1,2}$.

Hasta hace algunos años su tratamiento era médico o quirúrgico, dependiendo de muchas variables. El médico, con prolongadas quimioprofilaxis, para evitar las infecciones esperando la mejoría espontánea con el crecimiento del niño y , el quirúrgico mediante distintas técnicas las que buscaban reestablecer el túnel submucoso del uréter distal al llegar a la vejiga, para conformar el mecanismo valvular que evita el reflujo. Ambas con resultados similares en el largo plazo ${ }^{3}$.

En 1981 Matouschek introduce el tratamiento endoscópico ${ }^{4}$, el que posteriormente es popularizado por O’Donell y Puri en $1984^{5}$, quienes utilizando teflón (Politef), logran muy buenos resultados en el tratamiento de distintos grados de RVU. La polémica surgió en ese entonces respecto a la sustancia a inyectar, ya que ésta debía ser no antigénica, no migrar, perdurar en el tiempo y ser de fácil aplicación.

Fue así como surgieron distintas sustancias, el teflón nunca fue aprobado por la FDA, ya que en experimentos en animales se encontró que podía migrar, tanto a pulmón como a cerebro ${ }^{6}$. El colágeno se demostró como poco "durable", ya que producía alta tasa de recurrencia en el tiem$\mathrm{po}^{7}$. También se intentó con elementos autólogos como la grasa y el cartílago sin buenos resulta$\mathrm{do}^{8}$.

Hace 12 años aparece el Deflux, compuesto de microesferas de dextranomero en un gel de acido hialurónico, ambos componentes hechos de polisacáridos, basados en moléculas de azúcar. Las microesferas además tienen una dimensión de entre 80 y 250 micrones, lo que la hace no migrable y ambos componentes del Deflux son biocompatibles por lo que no producen efectos de rechazo, al parecer cumple con todos las cualidades para ser usado en el tratamiento endoscópico del $\mathrm{RVU}^{9,10}$

\section{MATERIAL Y MÉTODOS}

Desde el 2002 en la unidad de Urología del servicio de Cirugía Pediátrica del hospital de niños Dr. Exequiel González Cortés estamos uti- lizando Deflux para el tratamiento endoscópico de algunos reflujos vésicoureterales primarios, los casos han sido elegidos en forma aleatoria, principalmente dependiendo del hecho que los padres acepten el tratamiento, sabiendo que tiene un éxito menor que la cirugía abierta y que pudiesen conseguir parte del financiamiento del producto.

Analizamos los casos realizados entre 2002 y 2005 que tuvieran al menos 6 meses de seguimiento. Todos los pacientes tienen ecografia 4 semanas postoperatoria y uretrocistografia convencional o isotópica, a las 12 semanas. Un total de 25 pacientes cumplían éstos requisitos, de ellos se analiza edad, sexo, grado de reflujo, lateralidad, duplicidad y resultado del tratamiento en distintos grupos, después de una inyección de Deflux, pese a que en 2 pacientes se ha realizado una segunda inyección con buenos resultados. Se consideró tratamiento exitoso, cuando se comprobó con imágenes en su control la ausencia completa del RVU.

El procedimiento fue realizado a través de un cistoscopio con canal de trabajo recto 9,5 ó 12 French dependiendo de la edad y sexo, con video, quedando registradas las imágenes. La inyección de Deflux submucoso en el meato ureteral y algo por dentro de éste, se realizó a través de una aguja ad-hoc de 4 ó 5 french (Fig. 1), dependiendo del canal de trabajo del cistoscopio, con la técnica STING, registrando el volumen inyectado en cada uno de ellos, deteniendo la inyección al lograr una imagen satisfactoria tipo "volcán" a nivel del meato ureteral. (Fig. 2).

Todos los casos fueron ambulatorios, se realizo control ecográfico a las 4 semanas y se continúa la profilaxis hasta la realización de la cistografia 12 semanas postoperatorias.

\section{RESULTADOS}

De los 25 pacientes que entraron en nuestro estudio 11 eran bilaterales y 6 tenían doble sistema, de éstos últimos en dos existía reflujo a ambas unidades renales por lo que la muestra total está compuesta por 38 uréteres refluyentes.

21 pacientes eran de sexo femenino (84\%), todos se presentaron como infección urinaria, y en su estudio se diagnostico el reflujo de grado variable. La indicación quirúrgica fue por falla del 


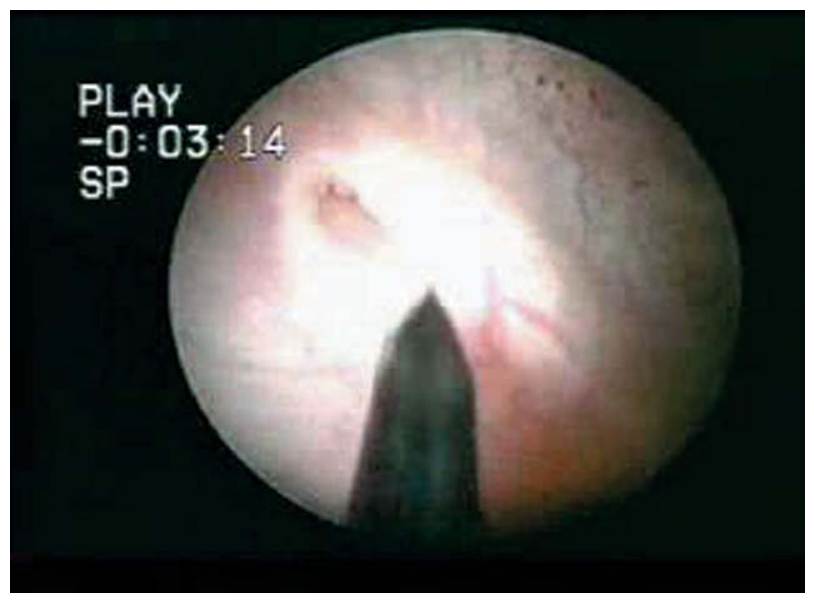

FIGURA 1

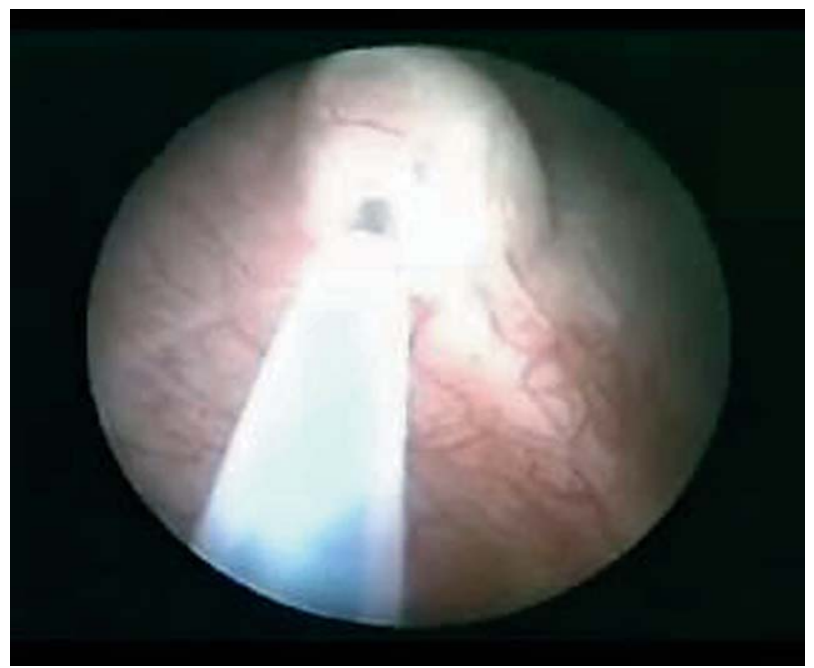

FIGURA 2

tratamiento médico, por infección intercurrente y/o mantención o empeoramiento del RVU durante su tratamiento de al menos 18 meses. La edad promedio de nuestros pacientes fue de 6,1 años, abarcando desde los 2 a los 13 años.

En cuanto a los grados de RVU, (Tabla 1) la mayoría son grados 3 (47\%), hay 6 uréteres con grado 1, todos son bilaterales, acompañando a grados mayores en su uréter contralateral, y hay un paciente con un reflujo grado 5 .

La cantidad de material inyectado fue variable entre $0,3 \mathrm{ml}$. a $1 \mathrm{ml}$. y no encontramos relación alguna entre el volumen inyectado y el éxito del procedimiento.

Del total de 38 uréteres inyectados, en 28 $(73,6 \%)$ desapareció completamente el reflujo y en 4 disminuyó de grado. Al analizar éste resultado
Tabla 1. Distribución inicial de pacientes

\begin{tabular}{lccc}
\hline Grado & Simple & Doble & Total \\
\hline I & 5 & 1 & 6 \\
II & 10 & 1 & 11 \\
III & 15 & 3 & 18 \\
IV & 1 & 1 & 2 \\
V & 1 & 0 & 1 \\
Total & 32 & 6 & 38 \\
\hline
\end{tabular}

nos encontramos con que en los dobles sistemas es donde obtuvimos resultados mas pobres, mejorándose solo 1 de 6 uréteres, e incluso en uno aparece reflujo contralateral, en cambio en los de sistema únicos encontramos un éxito de $85 \%$, y si son unilaterales como ocurrió en 10 de nuestros pacientes el éxito fue completo (100\%). En cuánto al análisis del resultado con los grados, también nos encontramos con variaciones, obteniendo un éxito de un $100 \%$ en los grados 1 y 2, bajando a solo $73 \%$ en los grados mayores. (Tabla 2).

Tabla 2. Resolución reflujo en sistema único

\begin{tabular}{lrc}
\hline Grado & & Resolución \\
\hline I & 5 & $5(100 \%)$ \\
II & 10 & $10(100 \%)$ \\
III & 15 & $11(73.3 \%)$ \\
IV & 1 & 1 \\
V & 1 & 0 \\
Total & 32 & $27(84,3 \%)$ \\
\hline
\end{tabular}

El seguimiento post operatorio fue de un promedio de 30 meses, (12-44 meses). No hubo complicaciones postoperatorias. En un caso apareció un reflujo de bajo grado en el uréter contralateral, el cual se está tratando en forma médica. Infección urinaria no febril se registró en 5 pacientes, 3 de los cuales eran del grupo que fallo y tenían reflujo asociado a un doble sistema. Solo en 2 pacientes se ha realizado una segunda inyección, siendo exitosa en ambos casos.

\section{DISCUSIÓN}

El RVU en niños es una patología potencialmente grave, por lo que debe tratarse. Desde hace algunos años se ha descrito el tratamiento 
endoscópico, el que ha ido adquiriendo mas adeptos en la medida de la aparición de "mejores" sustancias a inyectar, la cual debe ser no antigénica, durable, no migrar y de fácil aplicación, al parecer el Deflux reúne éstas condiciones por lo que se ha estado usando en forma creciente .

Sin duda existen otras sustancias inyectables de reconocida confiabilidad, nuestro equipo presentó hace 2 años un trabajo comparativo en mas de 50 pacientes inyectados con Colágeno, Macroplastique y Deflux, donde demostramos un éxito similar de éstas dos últimas sustancias, (alrededor de 80\%), nos decidimos por el Deflux, debido a su mayor disponibilidad en el mercado y su mayor facilidad de manejo, ya que no necesita una "pistola " para su inyección, gracias a su menor viscosidad.

Es bien reconocido el buen resultado con Macroplastique, incluso con reflujos de alto grado, el que llega a cerca de un $80 \%$ con una inyección, pero a un $100 \%$ de éxito con una tercera aplicación. Sin embargo la experiencia con el Deflux es mayor y sus resultados son similares $^{11,12,13}$. Respecto a la durabilidad en el tiempo, sabemos que hay trabajos que muestran mejoría con 10 años de seguimiento ${ }^{14}$.

El tratamiento endoscópico claramente tiene ventajas sobre el tratamiento quirúrgico, ya que es de fácil ejecución, con resultados alentadores y reproducibles, que día a día son mejores con éxitos cercanos al $100 \%{ }^{15}$. No requiere de hospitalización y registra una tasa de complicaciones muy baja ${ }^{16,17}$. La aparición de reflujo contralateral puede ocurrir al igual que en la cirugía abierta, como nos sucedió en un caso, está descrito en un $4,5 \%$ a $13 \%^{18}$, por lo que podría ser recomendable la inyección bilateral en aquellos uréteres en riesgo. En la actualidad agregando una hidrodistensión al uréter previo a la inyección se han visto resultados aun mejores ${ }^{19}$.

A la luz de resultados exitosos recientes en muchos centros se está utilizando el tratamiento endoscópico para el RVU como tratamiento de primera línea, incluso reemplazando al tratamiento médico, que es largo, tedioso y mal cumplido para muchos pacientes y sus padres, demostrándose una adherencia a la profilaxis incluso menor al 20\%. Además aprovecha de evitar la realización de frecuentes exámenes, y de exposición a múltiples radiaciones. Por otra parte los costos, si bien es cierto no son menores, al comparar con tratamientos prolongados o cirugías que requieren mayor estadía hospitalaria, también se ven disminuidos, aunque sea necesaria una segunda inyección

Es evidente el menor trauma que ocasiona el tratamiento endoscópico en nuestros pequeños pacientes, por lo que asociado a un buen resultado en la era de lo mínimamente invasivo, éste debiera convertirse en la primera elección del tratamiento del reflujo vésicoureteral pediátrico.

\section{CONCLUSIONES}

El RVU es una patología que debe ser tratada ya que puede llevar a la pérdida de función renal, el tratamiento clásicamente ha sido médico o quirúrgico, pero desde hace ya algunos años se ha popularizado el tratamiento endoscópico, gracias al hallazgo de sustancias adecuadas como el Deflux.

Presentamos nuestra experiencia con 38 uréteres inyectados, donde tenemos un éxito libre de reflujo con una inyección en 28 de ellos. Se obtuvieron mejores resultados en los de sistema único y unilaterales en donde todos se mejoran, contrastando éste éxito con la mejoría de solo 1 de los 6 uréteres con doble sistema. El procedimiento es rápido y seguro, no presentando complicaciones. Recomendamos el uso del tratamiento endoscópico con Deflux para el RVU primario en sistemas simples, dejando otras opciones para los dobles sistemas.

\section{REFERENCIAS}

1. Connolly LP, Zurakowski D, Connolly SA, Peters CA, Drubach LA, Cilento BG, et al. Natural history of vesicoureteral reflux in girls after age 5 years. J Urol. 2001;166 (6):2359-2363 .

2. Chertin B, Puri P. Familial vesicoureteral reflux. J Urol. 2003;169(5): 1804-1808.

3. Medical versus Surgical treatment of primary vesicoureteral reflux, Report of the International reflux study committee. Pediatrics. 1981;67(3):392-400.

4. Matouschek E. Treatment of vesicorenal reflux by transurethral teflon-injection (author's transl). Urologe A. 1981;20(5):263-264.

5. O'Donnell B, Puri P. Treatment of vesicoureteric reflux by endoscopic injection of Teflon. Br Med J (Clin Res Ed). 1984; 289(6436):7-9.

6. Steyaert H, Sattonnet C, Bloch C, Jaubert F, Galle P, Valla JS. Migration of PTFE paste particles to the kidney after treatment for vesico-ureteric reflux. BJU Int. 2000; 85(1): 168169. 
7. Caldamone AA, Diamond DA. Long term results of the endoscopic correction of vesicoureteral reflux in children using autologous chondrocytes. J Urol. 2001;165(6 Pt 2):2224-2227.

8. Frey P, Lutz N, Jenny P, Herzog B. Endoscopic subureteral injection for the treatment of vesicoureteral reflux in infants and children . J Urol.1995;154(2):804-807.

9. Heel RC, Morton P, Brogden RN, Speight TM, Avery GS. Dextranomer : A review of its general properties and therapeutic efficacy. Drugs. 1979;18(2): 89-102.

10. Stenberg A ,Lackgren G. A new bioimplant for the endoscopic treatment of the vesicoureteral reflux. Experimental and short term clinical results. J Urol. 1995;154(2 Pt 2): 800-803.

11. Elder JS, Diaz M, Caldamone AA, Cendron M, Greenfield S, Hurwitz R, et al. Therapy for Vesicoureteral Reflux: A Meta-Analysis. I. Reflux Resolution and Urinary Tract Infection. The Journal of Urology. 2006;175(2):716-722.

12. Chertin B, Puri P. Endoscopic Manegement of Vesicoureteral Reflux: Does It Stand the Testo of Time?. European Urology. 2002;42(6):598-606.

13. Keating MA. Role of periureteral injections in children with vesicoureteral reflux. Curr Opin Urol. 2005;15(6):369-373.

14. Van Capelle JW, de Haan T, El Sayed W, Azmy A.. The long-term outcome of the endoscopic subureteric implantation of polydimethylsiloxane for treating vesico-ureteric reflux in children: A retrospective analysis of the first 195 consecutive patients in two european centres. BJU Int. 2004;94(9):1348-1351.

15. Puri P, Chertin B, Velayudham M, Dass 1, and Colhoun E. Treatment of vesicoureteral reflux by endoscopic injection of dextranomer/hyaluronic Acid copolymer: preliminary results. J Urol. 2003;170(4 Pt 2):1541-1544; discussion 1544.

16. Vandersteen DR, Routh JC, Kirsch AJ, Scherz HC, Ritchey ML, Shapiro E, et al. Postoperative Ureteral Obstruction After Subureteral Injection of Dextranomer/Hyaluronic Acid Copolymer. The Journal of Urology. 2006;176(4 Pt 1):1593-1595.

17. Capozza N, Lais A, Nappo S, Caione P. The Role of Endoscopic Treatment of Vesicoureteral Reflux: a 17-year experience. The Journal of Urology. vol. 2004;172(4 Pt 2):1626-1628; discussion 1629.

18. Elmore JM, Kirsch AJ, Lyles RH, Perez-Brayfield MR, Scherz HC. New Contralateral Vesicoureteral Reflux Following Dextranomer/Hyaluronic Acid Implantation: Incidence and Identification of a High Risk Group. The Journal of Urology. 2006;175(3 Pt 1):1097-100; discussion 1100-1101.

19. Kirsch AJ, Perez-Brayfield M, Smith EA, Scherz HC The modified STING procedure to correct vesicoureteral reflux improved results with submucosal implantation within the intramural ureter. J Urol. 2004;171(6 Pt 1):2413-2416.

Correspondencia autor: Dr. José Manuel Escala A.

Unidad Urología Pediátrica. Hospital Exequiel González Cortes

Barros Luco 3301, San Miguel. Santiago- Chile

Tel: 56- 2- 4605307

E-mail autor: jmescala@clinicalascondes.cl

Información artículo: Original - Urología pediátrica

Trabajo recibido: diciembre 2006

Trabajo aceptado: mayo 2007 